\title{
A Plan for the Development and Demonstration of Optical Communications for Deep Space
}

\author{
J. R. Lesh \\ Communications Systems Research Section \\ L. J. Deutsch \\ TDA Technology Development Office \\ W. J. Weber \\ NASA Technology Program Office
}

\begin{abstract}
In this article, an overall plan for the development and demonstration of optical communications for deep-space applications is presented. The current state of the technology for optical communications is presented. Then, the development and demonstration plan is presented in two parts: the overall major systems activities, followed by the generic technology developments that will enable them. The plan covers the path from laboratory subsystems demonstrations out to a full-scale flight experiment system for the proposed Mars Communications Relay Orbiter mission.
\end{abstract}

\section{Introduction}

Piloted deep-space missions of the next century will potentially require downlink data rates on the order of $300 \mathrm{Mbps}$. Such rates would arise to support an aggregate of audio, high-resolution video, and instrument telemetry applications-even after compression. The first mission with such requirements is likely to occur on or before the year 2017 according to scenarios developed as part of the 1989 90-day study activity in support of a presidential lunar and Mars initiative.

Current deep-space missions have data rates of a few hundred kilobits per second. Hence, these anticipated new requirements represent an increase in downlink capability of at least three orders of magnitude. In order to effect that much of a performance increase, many new technologies will have to be assimilated by the Deep Space Net- work (DSN) as well as by spacecraft systems. One of the technologies that may be used to achieve this kind of performance improvement is optical communications.

Because of the large propagation loss resulting from beam divergence, returning high-rate data from deepspace vehicles to the Earth is an extremely difficult task compared to communication with Earth-orbiting satellites. Current outer-planet spacecraft use 4- to 5-m radio frequency (rf) antennas and 20-W rf output transmitters to return data, and the Earth reception systems use antennas as large as $70 \mathrm{~m}$ in diameter (often arrays of antennas are required). One way to reduce the loss from beam divergence is with the use of higher frequency carrier signals. This is the main reason that the DSN is currently planning to implement a Ka-band (32-GHz) communications capability. An even greater performance improvement is 
achieved by choosing still higher frequencies, such as those used in optical systems, if the corresponding technologies can be developed and assimilated. Of course, the precision with which such systems must point increases with the frequency as well, and this is one of the problems that must be solved for any such system.

Assuming that all the technological issues can be solved, it has been found that $300 \mathrm{Mbps}$ can be achieved on a Mars-to-Earth downlink by using a $10-\mathrm{W}, 0.8-\mu \mathrm{m}$ wavelength semiconductor laser (or array of lasers) together with a $1-\mathrm{m}$ transmitting telescope on the spacecraft. The ground receiving aperture would be $15 \mathrm{~m}$ in diameter and would not be required to focus the received energy any better than 100 times the diffraction limit for that frequency of light-i.e., it could be a photon bucket. These numbers are well within the expected technology evolution for this period of time.

In this article, a plan for the development and demonstration of optical-communications technology and systems capabilities is presented. The plan is a coordinated approach involving three NASA offices-the Office of Space Operations (OSO) Advanced Systems Office, the Office of Aeronautics and Exploration Technology (OAET) Information Sciences and IIuman Factors Division, and the Office of Space Science and Applications (OSSA) Communications Division-and is the result of 10 years of experience in the development of optical technologies and many previous planning activities.

In Section II, the major accomplishments of the past and the current activities in the optical-communications program are briefly reviewed. Next, the current technology development and system study activities are covered. The past and present activities form a basis for proposed activities of the future. The overall long-range plan, including major proposed flight experiment opportunities, are then discussed. This is followed by a description of the supporting technology development plan. The article closes with conclusions and prospects for the future of this promising technology.

\section{The Current Program}

A substantial number of technology developments, systems studies, and demonstration planning activities have been, or are now, in progress. A brief summary of those activities is presented here. A more thorough summary is given in the Appendix.

The Jet Propulsion Laboratory (JPL) deep-space optical-communications activity began over 10 years ago under OSO - formerly the Office of Space Tracking and Data Systems (OSTDS)--sponsorship with several theoretical studies of optical-communications channel capacity [1-4]. These led to the first experimental task, a laboratory demonstration of multiple bits per detected photon communication $[5 ; 6]$. Although somewhat restricted in its intended applications (to very low optical background environments), this demonstration established optical communications as a recognized entity for future deep-space planning.

A major impediment to the use of optical communications was the lack of reliable and efficient laser sources with adequate output power. For the first several years, the focus was on the development of semiconductor lasers and more specifically semiconductor laser arrays. Although monolithic laser-diode arrays are excellent candidates for moderate-range applications, they are incapable of providing the peak-power levels ( 1 to $5 \mathrm{~kW}$ ) required for many of the farther deep-space applications. They also exhibit unstable spatial mode output beams. For these reasons, other laser concepts were also considered.

The most promising candidate was the laser-diodepumped, neodymium-doped, yttrium-aluminum-garnet (Nd:YAG) laser. Unfortunately, the electrical-to-optical conversion efficiencies of such structures were extremely low (below 0.5 percent) at that time. Through a novel matched-cavity end-pumped design, such a laser was developed and demonstrated at JPL, achieving $100-\mathrm{mW}$ output with 8.5-percent overall conversion efficiency $[7,8]$. (Similar structures have now been used elsewhere to achieve efficiencies of 17 percent [9].) Using this design approach, a Nd:YAG laser module containing two laserdiode-array pumps and housed in a custom-designed titanium holder was later developed under OAET-formerly Office of Aeronautics and Space Technology (OAST) funding and successfully withstood space environmental testing (thermal and vibrational) [10].

Another area limiting optical communications is that of detectors. High-channel-efficiency signaling schemes for deep space require photodetectors that can respond to single-photon arrival events. This typically dictated the use of photomultiplier tubes. Unfortunately, these tubes suffer from being fragile, they have low quantumdetection efficiencies ( $<13$ percent), and they require voltage supplies of several thousand volts. To circumvent these drawbacks, a task was initiated to evaluate semiconductor avalanche photodiode detectors in a special cooled and overbiased mode. The concept requires cooling the device to about $200 \mathrm{~K}$ where the thermal generation of free carriers is sufficiently suppressed to permit biasing the detec- 
tor beyond its avalanche breakdown voltage. Avalanche triggering occurs when a photoelectron is released, thus clamping the applied voltage at the avalanche breakdown level. Using this configuration, single-photon detection at 25 to 40 percent quantum efficiency has been achieved [11].

Several subsystem-level technology developments are also in progress. An Integrated Optical Communication Test Bench (IOCTB) has been established for the design and evaluation of spatial acquisition and tracking techniques and a second demonstration system for heterodyne optical reception has been created. Limitations imposed by the Earth's atmosphere on communications performance are also being assessed. To evaluate the impact of atmospheric cloud cover, an Autonomous Visibility Monitoring (AVM) [12] program has been established; this program consists of three automatic telescope observatories. The observatories will be deployed at spatially diverse locations around the southwest United States, and will be used to characterize the joint visibility function of the atmosphere.

In order to support anticipated demonstrations (see Section III), planning and preliminary systems design are in progress for a 10-m-diameter optical reception facility to support optical-communications research and development. Preliminary design has also begun for a smaller (1-m-diameter telescope) transportable ground reception terminal to support several of the early flight demonstrations. The breadboard (BB) of an extremely small ( $<5$-kg mass) optical communications package called the Small Communications Optical Package Experiment (SCOPE) is also under development.

Finally, preparations are underway for a demonstration of uplink optical communications with the Galileo spacecraft during the Earth-gravity-assist phases. The demonstration, called GOPEX ${ }^{1}$, will use the spacecraft's imaging camera to receive uplink optical signals. This demonstration will be the first attempt to establish an optical communications link over deep-space distances.

\section{Long-Range Development and Demonstration Plan}

With this background, the long-range plan can be described. First, the overall long-range development, demonstration, and decision plan are discussed, focusing on the

\footnotetext{
${ }^{1}$ Galileo Optical Experiment (GOPEX), a proposal submitted to NASA by JPL (internal document), Jet Propulsion Laboratory, Pasadena, California, July 6, 1989
}

major demonstration package and support facility activities. This is followed by the generic technology development plan that will support these activities.

\section{A. Overall Plan}

The overall plan for optical-communications development is shown in Fig. 1. This section describes the plan beginning with a discussion of the space-segment developments. Following that, the support elements are described. Key decision points are flagged.

The plan aims for a full-scale experimental capability on the Mars Communications Relay Orbiter (MCRO) to be launched in 2003, the decision having to be made by 1998. (An alternative vehicle for the experimental package is the Mars Site Reconnaissance Orbiter, which is planned for launch that same year.) For planning purposes, it is assumed that the flight project start is 1998 , and delivery of the flight optical-communications equipment package for spacecraft integration is required by 2001 . Operational application of this technology is seen in the 2010 time period with a $300-$ Mbps link capability from Mars.

During 1990-91, the 5-kg SCOPE breadboard optical package will be completed. The SCOPE breadboard will be used in a series of laboratory and near-range groundto-ground demonstrations. The breadboard will consist of a $100-\mathrm{mW}$ laser diode, a $1-\mathrm{cm}$ beam diameter, and a quad avalanche photodiode tracking detector to track a Nd:YAG beacon laser signal. The breadboard is being built using designs that could permit future space qualification.

In 1991, development of a Space Technology Demonstration System (STDS) will commence. It will have two interchangeable laser modules: one a diode laser and the other a $250-\mathrm{mW}$ diode-pumped Nd:YAG laser. The telescope aperture will be $20 \mathrm{~cm}$ in diameter, making the system a prototype for possible future lunar applications. It will also have the acquisition and tracking fine-pointing equipment necessary to demonstrate microradian beam pointing. The STDS would be flown on the space shutthe in 1995. The STDS development is also consistent with the time frame for a mission enhancement experiment on the proposed Lunar Observer (LO) spacecraft. An assumed LO launch date is shown for reference. The decision date is 1994 . The STDS would be used for spaceto-ground demonstrations in the 1997-2003 period, again supported by the transportable ground terminal. If flown on the space shuttle, space-to-space demonstration links could also be demonstrated if other spaceborne terminals are available (e.g., the Laser Communications Transceiver [LCT] being studied by NASA Goddard Space Flight Cen- 
ter as a Space Station attached payload or the Semiconductor Laser Intersatellite Link Experiment [SILEX] terminal being developed by the European Space Agency). If flown on a lunar mission, a data rate of $100 \mathrm{Mbps}$ could be transmitted back from the Moon to a 1-m receiving aperture.

The engineering model (EM) development for the MCRO payload is scheduled to commence in 1996 . It will draw from the STDS heritage, but will use a $60-\mathrm{cm}$ telescope and at least a $2-W$ laser. The EM will be evaluated in 1998-99, after which development of the flight unit can commence. The MCRO package can support 10 - to 60 -Mbps data return from Mars to a 10 -m-diameter receiver (depending on the planetary range). Primary demonstration activities would occur in the 2004-2008 period.

To support the spaceborne demonstration activities, a companion development program is planned for groundbased capabilities. It is assumed that the GOPEX demonstration and the deployment of the AVM observatories continue on schedule. To provide reception support to the STDS, a small transportable optical terminal is planned. It will consist of a semitrailer-based 1-m-diameter receiving telescope, with a smaller $(10-\mathrm{cm})$ uplink telescope. The telescope tracking will be sufficiently fast to track lowaltitude satellites (e.g., the shuttle). All the lasers, optics, and processing electronics required for the terminal will be housed in the trailer. This terminal will serve both as a system test bed for a follow-on $10-\mathrm{m}$ facility, as well as an engineering model for a lunar-support network station. Completion of the terminal is scheduled for 1993 so that adequate shakedown can occur before supporting the STDS demonstrations. The primary demonstration period for this terminal is 1994-2001. It will also be very useful for certifying future ground reception sites, and it will collect much useful information on the operational noise (light) background.

By 1997, the construction of a facility for opticalcommunications research and development is expected to begin, assuming approval of the Construction of Facilities (C of F) proposal by 1995. The Deep-Space Optical Reception Antenna (DSORA) will consist of a $10-\mathrm{m}$ diameter segmented aperture with an integral sunshield that permits small Sun-Earth-probe (SEP) angle viewing. Fraunhofer filters [13] will be included to cut down the telescope's susceptibility to solar background. A smaller (1-m) telescope of high surface quality will be boresight aligned with the main telescope and will be used for both uplink transmissions (command and ranging) and astrometric tracking of signals from spaceborne platforms against the stellar background. Upon completion in 2000, it will be available for preliminary demonstrations using the STDS, followed by experiment support for the MCRO package. Unlike the 1-m transportable system, DSORA will allow high-rate demonstrations with spacecraft similar to those with the expected operational capabilities. It will also serve as a prototype for future ground or Earth-orbiting terminals.

In 1999, planning activities for a future Mark 1 optical reception network are expected to commence, followed by development of the net beginning in 2004, the NASA decision to go ahead having been made by 2002 . This network would meet the needs of piloted Mars missions. It will be in place early enough to be used on at least one Mars robotic mission prior to its first use with a piloted mission.

\section{B. Technology Plan}

A number of generic technologies are needed to realize the objectives of the overall plan. The development plan for these technologies is shown in Fig. 2.

The first item is the development of laser transmitters for flight systems. The initial STDS transmitter module, based on a laser-diode source, will be developed by 1993 . It will output at least $50 \mathrm{~mW}$ of power and be modulated at up to $10 \mathrm{Mbps}$. This will be followed by the development of two additional laser transmitters for the STDS: one a laser-diode-based unit with 100 -Mbps datarate capability and the other a Nd:YAG laser for lower rate deep-space applications. The 60 -Mbps transmitter for the MCRO transceiver will be developed by 1998 , followed by a 300 -Mbps transmitter for later martian applications.

The issue of optical uplinks for command and ranging purposes will be addressed beginning in FY94. Also, the problem of an emergency uplink needs to be solved. If the emergency link is to be optical, then both high-power (1- to $10-\mathrm{kW}$ ) lasers for the ground and omnidirectional optical receiving telescopes for the spacecraft will need to be developed.

Acquisition, tracking, and pointing (ACK/TRK/PTG) are extremely important functions for an optical-communications application. From 1990 to 1993, an ACK/TRK/ PTG system for the STDS will be developed. It will track a point-source (laser) beacon signal and demonstrate $1-\mu$ rad pointing. By 1995 , the system will be upgraded to 300-nrad pointing. For the MCRO experimental package, a 130-nrad ACK/TRK/PTG technology will be developed by 1998 . Unlike the earlier pointing systems, it will derive its pointing reference not from an uplink beacon laser, but 
from the solar-illuminated Earth image. This eliminates schedule-conflict problems that arise between the beacon laser and the visibility to the Earth reception station when the Earth-to-spacecraft propagation time becomes large. By 2004, an improved 50-nrad pointing system will be developed for the future $300-\mathrm{Mbps}$ system, assuming success of the 130-nrad system.

Telescopes that are rigid, thermally stable, and lightweight will be required for space use. The development of a space-qualified optical-communications telescope will be accomplished by FY95, with subsequent evaluation by FY96. The telescope will have a primary aperture of $60 \mathrm{~cm}$ and serve as a prototype for the MCRO package telescope. Following this, a 1-m diameter telescope will be developed for the later $300-\mathrm{Mbps}$ system. Also shown is the development cycle for the STDS telescope.

Optical "benches" and other structural elements that are rigid, thermally stable, and lightweight are required for optical-communications flight systems. A breadboard optical bench will be fabricated from graphite-epoxy during FY90 and will be evaluated for its thermal and structural stability. A follow-on proof-of-concept bench will be designed and fabricated by 1992; this bench will include optical-mount and bench-attachment techniques. Beyond 1992 and up through the beginning of the MCRO engineering model development in 1995, this task will develop and evaluate other structural elements made of advanced composite materials (e.g., support struts, sunshields, and enclosures).

Detector development will also continue, particularly for Earth-vicinity reception systems. Suitable detectors will be developed for the 10-Mbps and 100-Mbps systems for the STDS. Detectors appropriate for 300-Mbps operation will be developed by 1999 .

Earth-reception systems must also filter out much of the unwanted background light since it results in interfering noise at the detector. Initially, these filters will be standard multidielectric interference filters. Later, more narrow Fraunhofer filters [13] tuned to spectral shadows in the solar spectrum will be developed, followed by even more narrow (fractions of an angstrom) atomic-resonance filters. Both the detectors and the filters will be evaluated at the systems level using the transportable ground terminal and then used in the DSORA.

One of the serious concerns for optical communications is the ability of the optical system to reject stray background light. This is extremely important in ground-based systems looking through the atmosphere. However, a similar concern exists for spacecraft applications when the
Earth appears close to the Sun. Techniques for reducing background light include bafling, low-scatter secondary support structures, sunshades, and selective optics coatings. The goal of the background-mitigation task is to reduce typical system susceptibility 50 percent by 1995 , 85 percent by 1998 , and 95 percent by 2000 . These improvements will be worked into the designs of the STDS and MCRO flight packages, assuming these are approved.

Another way to essentially eliminate the effects of bachground light is to use coherent optical heterodyne (as opposed to photon-counting) detection techniques. Colserent reception systems filter out the corrupting noise signals at a much more narrow intermediate-frequency signal, rather than at the full optical carrier frequency. Coherent systems can also provide very precise optical Doppler signals for navigational tracking. However, heterodyne processing requires much more precise optical surfaces and much more stringent optical-signal alignment than direct photon-detection systems. In order to evaluate the benefits and disadvantages of this technology, two activities are also included for coherent detection of optical signals. These, in conjunction with the other elements of the plan, will permit an informed decision on the appropriate reception technology for the later 300-Mbps application. The first activity involves the heterodyne detection of signals, initially with low-rate modulation (100 kbps), and then later at a higher rate (100 Mbps). This will be followed by the development of a brassboard heterodyne optical receiver assembly. The second activity is the development of a coherent optical transponder. The architecture of such a transponder will be developed by 1994, with design and laboratory evaluation occurring in 1997 and 1999 , respectively. Flight-qualifiable coherent transponder development will commence in the early years of the next century, assuming success of the earlier demonstrations.

Definition of the 10-m DSORA station will continue in order to provide timely inputs to the Construction of Facilities 5-year plan and appropriate program submissions for a 1997 start of construction. Technical support will continue thereafter for the detailed definition of the additional equipment used in the facility, as well as the development of experimental plans for use of the station when it is completed.

The AVM deployment and data-collection program will be augmented with other data sources, especially satelliteacquired data, and a set of optical ground-based reception weather models will be generated (with annual updates).

Finally, the planning and subsequent technology developments needed for the development of the Mark 1 optical 
reception network will commence in 1999. The technology cutoff date for this network will be in 2004, with work after that date going into evolutionary development and en liancements. This network is expected to provide highdata-rate coverage for the high-intensity martian exploration activities and for other future deep-space missions as well. Major questions, such as: Will the network be space-based or ground-based?, Will the network support lunar exploration and if so how?, and What additional technology developments are required or desired? will be answered by that time based on the prior technology development and demonstrations.

\section{Conclusions}

In this article, an overall plan for the development and demonstration of optical communications for deep-space applications was presented. The plan builds on the foundation established by the past 10 years of NASA technology development and systems study activities at, or sponsored by, JPL. Past and currently ongoing activities were described first. Then the development plan, consisting of both systems demonstration level and supporting technology portions, was presented. The plan covers the path from laboratory subsystem demonstrations out to a full-scale flight-experiment system on the Mars Communication Relay Orbiter mission and selected technology developments needed for an eventual 300-Mbps link from Mars. The path includes technology demonstrations on a Spaceborne Technology Demonstration System. Key decision points were flagged. Optical communications are expected to provide many capabilities complementary to the currently used rf communications, and to enable many of the more ambitious data communications scenarios of the Space Exploration Initiative. 


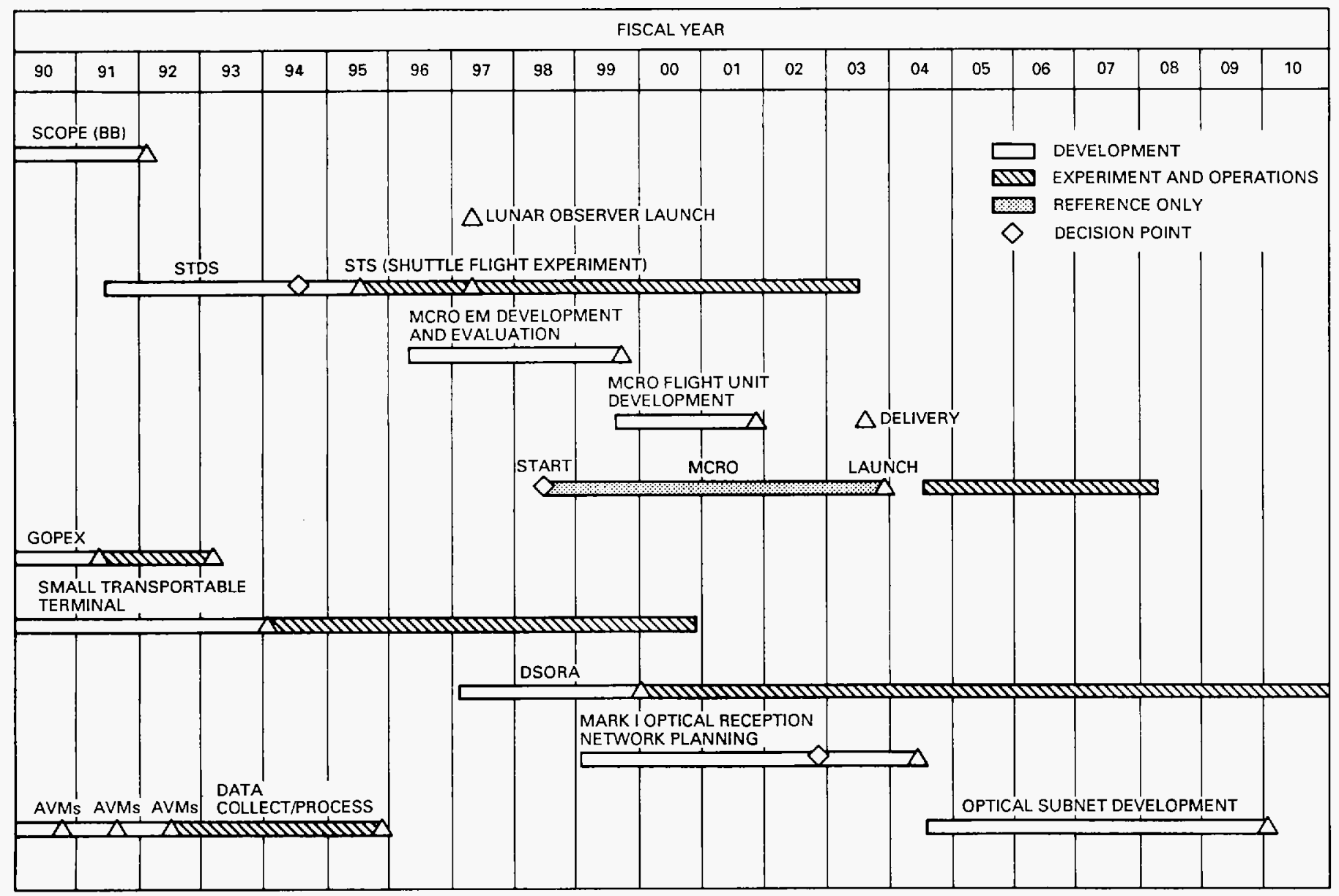

Flg. 1. Deep-space optical-communications long-range plan. (Shaded entry is shown for reference.) 


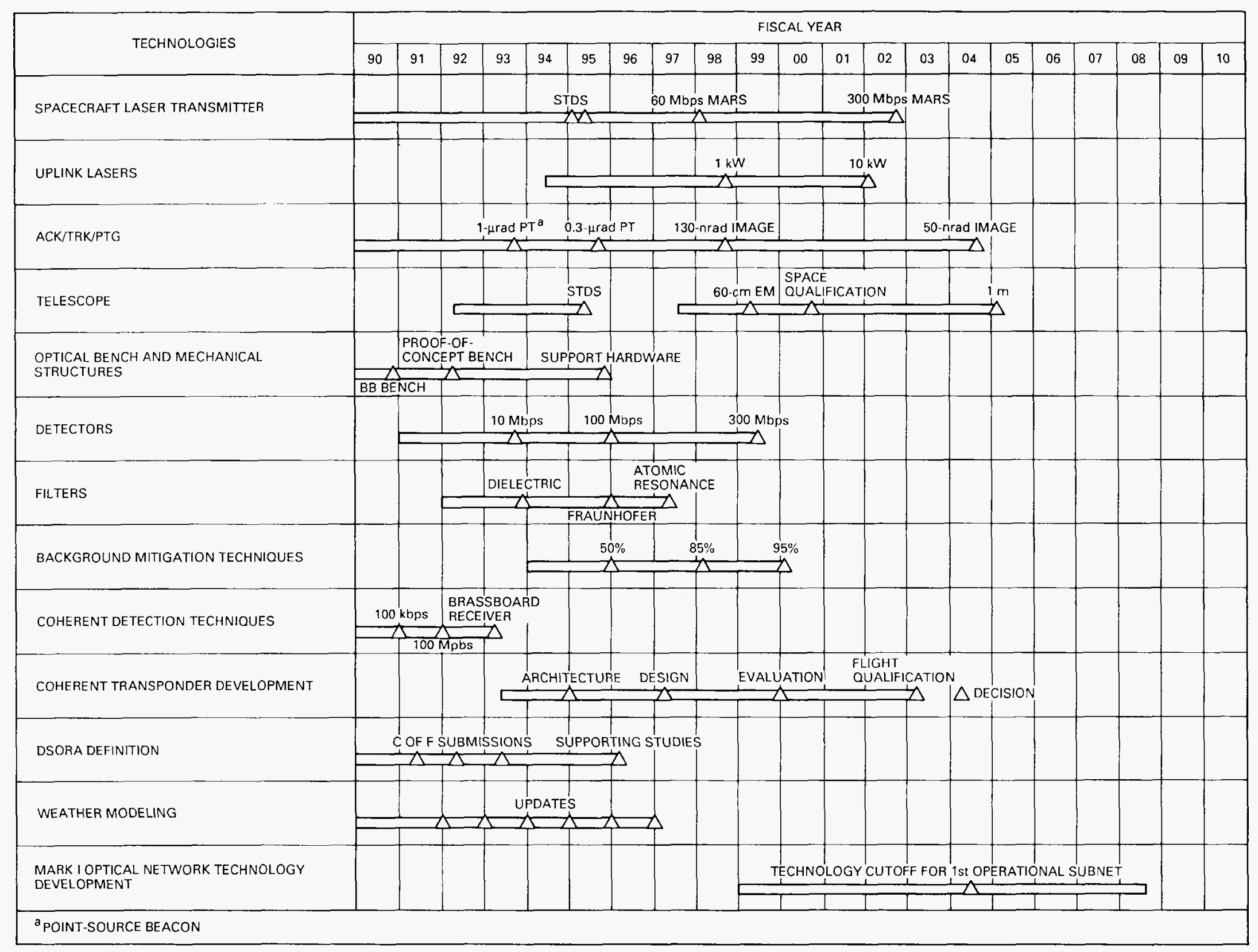

Fig. 2. Technology development plan. 


\section{Appendix \\ Expanded Summary of Current Activities}

Current optical-communications research and development activities are directed at reducing the mass, power consumption, cost, and risk of key components, as well as characterizing the effects of the atmosphere on groundbased reception options, planning for flight system demonstrations, and studying options for the Earth-vicinity reception network infrastructure.

\section{A. Key Component Technologies}

Laser development work in past years concentrated on power-efficient generation of light. Current research focuses on the modulation and frequency conversion of the light. These functions must be integrated into the laser cavity structure to preserve the efficiency of optical-signal handling. A diode-pumped Nd:YAG laser cavity with an acousto-optic Q-switch modulator and an intracavity frequency doubler for producing $0.53-\mu \mathrm{m}$ (green) light has been designed under OAET sponsorship. The laser will have a pulse rate up to $25 \mathrm{kHz}$ (corresponding to pulseposition modulation data rates up to $200 \mathrm{kbps}$, depending on coding assumptions) and produce an average output power of $0.25 \mathrm{~W}$. This design will later be extended to the 2- $W$ region.

One of the largest consumers of mass and power in an optical-communications system is the spatial acquisition, tracking, and beam-pointing subsystem. For this reason, OAET has sponsored the development of an Integrated Optical Communications Test Bench (IOCTB). This test bench consists of a telescope, steering optics, filters, photodetectors, and other optics that would be encountered in an optical-communications flight package, and is being used for the development of spatial acquisition and tracking algorithms and mechanisms. The present emphasis is on the acquisition and tracking of a point-source beacon: but will soon be modified to develop techniques for operating with spatially extended (non-point-source) beacon signals, such as the solar-illuminated Earth image. The test bench will also be used to simulate spacecraft vibrational dynamics, and to evaluate susceptibilities to, as well as mitigation techniques for, st.ray light.

Optical-communications subsystems require fairly precise alignment of the optical elements, and the structure supporting these elements must retain these alignment tolerances over the life and temperature cycling of the mission. Other national and foreign optical-communications programs have achieved these requirements with metal- lic optical benches that are temperature controlled (often to a fraction of a degrce) to preserve their dimensional stability $[14,15]$. Through an OSSA Communications Division program, the Mechanical Systems Division at JPL is developing optical-communications bench technology, which uses advanced composite materials such as graphite epoxy and carbon-carbon. A breadboard bench assembly is currently being designed that has a mass comparable to that of beryllium, but retains dimensional stability over typical temperature cycles passively (without an active temperature-control system). Such a benclı will save a substantial amount of mass and power associated with the heaters, control system clectronics, and heat radiators required for current metallic benches.

Although direct detection of optical signals is by far simpler to implement and will be used for most of the earlier experiments and operational systems, there are also key applications where the use of heterodyne optical detection is desired or required. These include highbackground-light applications, such as solar missions or spacecraft trajectories where the Sun is directly behind the vehicle, as well as situations where precise Doppler tracking is desired, such as low-altitude orbital missions. (Optical signals are essentially immune to the effects of chargedparticle fluctuations encountered in the solar wind.) A key ingredient in such systems is a narrow-linewidth frequencystabilized laser. For several years, JPL has supported Lightwave Electronics Corp. through the Small Business Innovative Research (SBIR) program in the development of frequency-stabilized nonplanar ring oscillator (NPRO) lasers. These devices are diode-pumped $\mathrm{Nd}: \mathrm{YAG}$ lasers that produce unidirectional ring lasing in a specially designed block of $\mathrm{Nd}$ :YAG material [16]. The isolation from optical feedback afforded by the ring structure enables very stable temporal oscillation. The free-running linewidths of these lasers are 5 to $6 \mathrm{kHz}$ (the carrier frequency is $300 \mathrm{THz}$ ). It is now understood that this is dominated by $1 / f$ flicker noise, which can be tracked out, leaving a residual linewidth of about $0.2 \mathrm{~Hz}$ [17]. A multiyear contract is also in place with Stanford University through the University Space Engineering Research Program to investigate methods of stabilizing and using such lasers.

As a result of the Lightwave Electronics SBIR contract, several of the frequency-stabilized NPRO lasers were delivered to JPL. These are being used in an OSSA-sponsored program to set up optical heterodyne receiver demonstrations. The first of these demonstrations is a low-rate 
(<1-Mbps) receiver using intensity-modulated binary pulse-position modulation (PPM). A follow-on demonstration is a higher-rate (100-Mbps) receiver that will use binary phase modulation. The low-rate demonstration has been set up using general-purpose instrumentation for intermediate-frequency (IF) signal processing and has demonstrated phase-locking of the local oscillator laser to the transmit laser. A custom-designed IF signal processor is being built and will be installed soon. The high-rate demonstration is awaiting a high-rate phase modulator, which is currently under development. Development of optical heterodyne-reception techniques is one step toward the eventual demonstration of a coherent optical transponder.

\section{B. Characterization of Atmospheric Effects}

One of the major future decisions is whether the operational reception of optical signals will initially be accomplished from Earth orbit or on the ground. Also, should the decision be made to deploy a ground-based network, there is a major need to characterize the reception performance. Both of these are critically dependent on having an extensive probabilistic model for the effects of the atmosphere, especially the effects of cloud cover. To obtain such statistics, three Autonomous Visibility Monitoring (AVM) observatories have been procured and will be set up with OSO Advanced Systems Office funding at spatially diverse locations around the southwestern U.S. [12]. Each observatory consists of a computer-controlled telescope, an internal star catalog, photodetectors, an observatory controller, data storage, and a telephone modem for communication with a central computer at JPL. The observatory equipment is housed in a roll-off roof dome that operates in conjunction with a set of weather sensors. The observatory telescope will automatically track and measure the light intensity level of a series of stars, at night as well as at daytime, unless foul-weather conditions are sensed. By deploying three such observatories at spatially diverse locations, all tracking the same star simultaneously, it will be possible to obtain joint visibility statistics of the atmospheric conditions. By analyzing data from a single station, marginal (one-station) weather statistics will also be available. Once collected, these data will be integrated with existing weather data, abtained primarily from satellite weather observations, and used along with atmospheric transmission software tools developed by other government agencies (primarily the DoD) to establish and maintain an optical weather model. The first of the AVM observatories is currently installed at $\mathrm{JPL}$ for test purposes, and will be deployed to a field location soon. The other two AVM stations are completed and will soon be delivered to JPL for evaluation. This program is supported by OSO.

\section{Reception Infrastructure and Flight Experiment Planning}

For the last several years, the OSO Advanced Systems Office has sponsored studies to define and develop the supporting rationale for an optical-reception facility for optical-communications research and development. Initial work concentrated on the survey of existing telescope facility cost and performance data, and resulted in a parametric cost-versus-performance and size model [18]. From this, choices were made on the size and surface quality of the telescope which kept the cost below the knee of the costperformance curve. This study resulted in the selection of a 10-m-diameter telescope, using a segmented primary aperture with a $2-\mu \mathrm{m}$ surface accuracy. A novel integral sunshield, which permits small ( $<10 \mathrm{deg}$ ) Sun-Earth-probe viewing while precluding direct sunlight from striking the primary mirror, was also formulated and analyzed [19]. Construction of Facilities proposal packages have been assembled during the past two years and design activities in the Ground Antennas and Facilities Engineering section have resulted in a bottom-up cost estimate that is within 10 percent of the parametric estimate. Definition work is still in progress, and is augmented by selected technology developments, including a contract with New Mexico State University to investigate diode-laser-pumped atomic resonance filters for high levels of background light rejection.

Several proposed flight-experiment packages have also been studied for optical communications, most of which have targeted the Cassini mission to Saturn. The original study was funded by OSO and was concerned with a mission-enhancement system for encounter [20]. Unfortunately, its $50-\mathrm{kg}$ mass was more than the mission could accommodate. This led to a series of OAET-funded reduced-size designs, finally ending with a very minimal Small Cassini Optical Package Experiment (SCOPE), which would have a mass of only $5 \mathrm{~kg}$ and be used for demonstration during the Earth-flyby encounter. A breadboard SCOPE package design was initiated. Unfortunately, the need to conserve mass on this particular spacecraft is very high and precludes flying even this experiment at this time. However, the SCOPE breadboard package is being developed and will be used for a series of laboratory and local field-level tests.

Finally, preparations are in progress through the OSO Advanced Systems Office for an optical communications demonstration with the Galileo spacecraft during its two Earth encounters for Earth-gravity assist. The Galileo Optical-communication using an Earth-based Xmtr (GOPEX) demonstration involves transmitting signals at power levels of from 7 to $20 \mathrm{~W}$, at both 1.06 - and $0.532-\mu \mathrm{m}$ 
wavelengths, from a commercial Nd:YAG laser through the 24-in.-diameter telescope at JPL's Table Mountain Observatory to the spacecraft. The imaging camera on board Galileo will be used as the optical detector. By scanning the camera past the Earth while sending pulse-modulated signals from the ground-based laser, the temporally modulated light beam is converted to a spatially modulated image on the camera's focal plane. The resulting camera image will then be transmitted to the ground via the standard rf link for processing.

The demonstration, which will be the first deep-space (beyond-lunar) optical-communications experiment ever, will be used to verify uplink pointing capabilities, to eval- uate the environment of a deep-space spacecraft for reception of uplink optical signals, and to verify opticalcommunications performance prediction models. A bestefforts demonstration will be conducted during the first Galileo-Earth encounter (December 1990) with the prime demonstration occurring in December 1992 at the second Earth encounter. Calibration tests of the uplink transmission system will be conducted both before and after the GOPEX demonstration using the Laser Geodynamic Satellite (LAGEOS) corner-cube-array-reflector satellite, and the Strategic Defense Initiative Organization (SDIO) Low-power Atmospheric Compensation Experiment ( $\mathrm{AACE}$ ) satellite. The latter is an orbiting target board instrumented to measure incident optical intensities. 


\section{References}

[1] J. R. Pierce, E. C. Posner, and E. R. Rodemich, "The Capacity of the Photon Channel," IEEE Transactions on Information Theory, vol. IT-27, no. 1, pp. 6177, January 1981.

[2] R. J. McEliece, "Practical Codes for Photon Communication," IEEE Transaclions on Communications, vol. IT-27, no. 4, pp. 393-398, July 1981.

[3] S. A. Butman, J. Katz, and J. R. Lesh, "Bandwidth Limitations on the Noiseless Optical Channel Capacity," IEEE Transactions on Communications, vol. COMM-30, pp. 1262-1264, May 1982.

[4] J. R. Lesh, "Capacity Limit of the Noiseless, Energy-Efficient Optical PPM Channel," IEEE Transactions on Communications, vol. COMM-31, no. 4, pp. 546-548, April 1983.

[5] J. R. Lesh, J. Katz, II. II. Tan, and D. Zwillinger, "2.5 Bit/Detected Photon Demonstration Program: Description, Analysis and Phase 1 Results," TDA Progress Report 42-66, vol. July-September 1981, Jet Propulsion Laboratory, Pasadena, California, pp. 115-132, December 15, 1981.

[6] J. Katz, "2.5 Bit/Detected Photon Demonstration Program: Phase II and III Experimental Results," TDA Progress Report 42-70, vol. May-June 1982, Jet Propulsion Laboratory, Pasadena, California, pp. 95-104, August 15, 1982.

[7] D. L. Sipes, "Highly Efficient Neodymium:Yttrium Aluminum Garnet Laser EndPumped by a Semiconductor Laser Array," Appl. Phys. Lett., vol. 44, no. 2, pp. 74-76, July 15, 1985 .

[8] D. L. Sipes, "Method and Apparatus for Efficient Operation of Optically Pumped Laser," U. S. Patent 4,710,940, Washington, D.C., December 1, 1987.

[9] W. J. Skrlac and H. P. Kortz, "High Power, High Frequency Neodymium Laser End-Pumped by a Single-Stripe Laser Diode," Proceedings of the LEOS 88, Santa Clara, California, paper EL1.2, November 1988.

[10] H. Hemmati and J. R. Lesh, "Reliability Testing of a Diode-Laser-Pumped Nd:YAG Laser and a Set of Diode Laser Arrays," Proceedings of SPIE OE Lase 89, paper 1059-22, January 1989.

[11] D. L. Robinson, and B. D. Metscher, "Photon Detection with Cooled Avalanche Photodiodes," Appl. Phys. Lett. vol. 51, no. 19, pp. 1493-1494, November 9, 1987.

[12] K. A. Cowles, "A Visibility Characterization Program for Optical Communications Through the Atmosphere," TDA Progress Report 42-97, vol. JanuaryMarch 1989, Jet Propulsion Laboratory, Pasadena, California, pp. 221-225, May 15, 1989.

[13] E. L. Kerr, "Fraunhofer Filters to Reduce Solar Background for Optical Communications," Optical Engineering, vol. 28, no. 9, pp. 963-968, September 1989.

[14] Opto-Mechanical Subsystem, Critical Design Revicwat Perkin Elmer Corp., MIT Lincoln Laboratory Contract BX-1806, Perkin Elmer, Danbury, Connecticut, October 6, 1987.

[15] R. Dumas and B. Laurent, "System Test Bed for Demonstration of Optical Space Communications Feasibility," Proceedings of SPIE OE Lase 90, Los Angeles, California, pp. 398-411, January 1990. 
[16] T. J. Kane and R. L. Byer, "Monolithic Unidirectional Single-Mode Nd:YAG Ring Laser," Opt. Lett., vol. 10, no. 2, p. 65, February 1985.

[17] C. C. Chen, M. Win, W. K. Marshal, and K. Shaik, "Coherent Link Demonstration for Space Communication," OSA Annual Meeting, Orlando, Florida, paper THT10, October 17-20, 1989.

[18] D. L. Robinson and J. R. Lesh, "A Cost-Performance Model for Ground-Based Optical Communications Receiving Telescopes," Proceedings of SPIE OE Lase 89, paper 756-20, pp. 130-134, January 1987.

[19] E. L. Kerr, "Architectural Design of a Deep-Space Optical Reception Antenna," submitted for publication in Optical Engineering.

[20] S. G. Lambert, Design and Analysis Study of a Spacecraft Optical Transceiver Package, Final Report-JPL Contract 957061, McDonnell Douglas Corp., St. Louis, Missouri, August 19, 1985. 